\title{
Dynamics of A Re-Parametrization of A 2-Dimensional Mapping Derived from Double Discrete Sine-Gordon Mapping
}

\author{
La Zakaria \\ Department of Mathematics, \\ Faculty of Mathematics and Natural Sciences, Universitas Lampung, Indonesia. \\ Corresponding author: lazakaria.1969@fmipa.unila.ac.id \\ Johan Matheus Tuwankotta \\ Analysis and Geometry Group, \\ Faculty of Mathematics and Natural Sciences, Institut Teknologi Bandung, Indonesia. \\ E-mail: theo@dns.math.itb.ac.id
}

(Received June 24, 2019; Accepted November 14, 2019)

\begin{abstract}
We study the dynamics of a two dimensional map which is derived from another two dimensional map by re-parametrizing the parameter in the system. It is shown that some of the properties of the original map can be preserved by the choice of the re-parametrization. By means of performing stability analysis to the critical points, and also studying the level set of the integrals, we study the dynamics of the re-parametrized map. Furthermore, we present preliminary results on the existence of a set where iteration starts at a point in that set, in which it will go off to infinity after finite step.
\end{abstract}

Keywords- Re-parametrizing, 2-dimensional mapping, Generalized double discrete sine-Gordon, Integral.

\section{Introduction}

Arguably, one of the most important and general integrable maps is known in the literature as the Quispel-Roberts-Thompson map (QRT). It is a two-dimensional map depending on 18 parameters. The QRT map is closely related to so called soliton equations (Quispel et al., 1988; Quispel et al., 1989). More recent studies have focused on generalizations of QRT maps. One of them was proposed by Joshi and Kassotakis (2019). Main result of their paper is a new connection between two major theories that generalize QRT maps. They provide a new formulation of QRT involutions in terms of Hirota derivatives and discover conditions under which each involution can be factorized into two further involutions.

The sine-Gordon equation is a partial differential equation, which is known to have soliton solutions; hence, it is a soliton equation (Quispel et al., 1991). Discretizations of the sine-Gordon equation have been done in various ways (Quispel et al., 1988; Quispel et al., 1989; Quispel et al., 1991). The reduction of the sine-Gordon equation to a two dimensional ordinary difference equations using a standard staircase (see Van der Kamp and Quispel, 2010 for the method) is known as being a special case of the celebrated QRT map. Recently, Celledoni et al. (2019) have studied a new systematic approach for calculating the preserved measures and integrals of a rational map in which a two-dimensional sine-Gordon (standard) map was chosen as an example (see Celledoni et al., 2019 §4.3 Example 4).

Generating a new family of mapping from a known one is an interesting topic to study. A piece-wise linear map from a known integrable map by using the ultra discretization technique is 
International Journal of Mathematical, Engineering and Management Sciences

Vol. 5, No. 2, 363-377, 2020

https://doi.org/10.33889/IJMEMS.2020.5.2.030

generated by Tuwankotta et al. (2004). The number of independent integrals are preserved by the transformation which implies that the integrability is preserved. In our article (Zakaria and Tuwankotta, 2016) a straight-forward generalization by adding parameters in the Lax pair of the ordinary discrete sine-Gordon partial difference equation has done. By using the standard staircase method, the resulted equation is then reduced to system of ordinary difference equations (see Van der Kamp et al., 2007 for the method). Note that this generalized sine-Gordon system is also analyzed in (Duistermaat, 2010).

Another novel method to introduce a new class of discrete systems from an integrable discrete system, is by introducing the concept of dual (Quispel et al., 2005). This works beautifully for a single discrete equation, although the resulting equation may not be new nor integrable. This idea of dual is extended to system of discrete equations in (Tuwankotta et al., 2019). The latter is interesting in the sense that the method proposed there produces in general more than one system.

Roberts et al. (2002) constructed a new family of mapping by interchanging the parameter and the integral. For example, consider a discrete dynamical system in $\mathbb{R}^{n}$ which is denoted by: $\mathbf{x}^{\prime}=\mathbf{f}_{\lambda}(\mathbf{x})$, where the prime denotes the upshift, $\mu$ is a parameter in the system, and $\mathbf{f}_{\mu}: \mathbb{R}^{n} \rightarrow \mathbb{R}^{n}$. We assume that the exists a smooth function $G: \mathbb{R}^{n} \times \mathbb{R} \rightarrow \mathbb{R}^{n}$ such that: $G\left(\mathbf{x}^{\prime}, \mu\right)=G(\mathbf{x}, \mu)$, which is called an integral for the system. Suppose that we can solve the equation $G(\mathbf{x}, \mu)=0$ for $\mu(\mathbf{x})$. Then by substituting this solution to $f_{\mu}(\mathbf{x})$ (and call it $g(\mathbf{x})$ ), we derive a new discrete system: $\mathbf{x}^{\prime}=g(\mathbf{x})$, with integral: $\mu(\mathbf{x})$.

We will follow this technique and apply it to a generalized sine-Gordon equation. The aim of this paper is to show a number of properties of the new mapping (after re-parameterization) and to compare them with the original mapping. Furthermore, the qualitative behavior of the new mapping is studied by means of obtaining fixed points and their stability, and also the base points.

The outline of this paper is the following. In Section 2, a system of first order difference equations derived from generalized double discrete sine-Gordon ( $\Delta \Delta$ sine-Gordon) equations is formulated by restricting to traveling wave solution. In Section 3 a new integrable mapping derived from $\Delta \Delta$ sine-Gordon mapping by interchanging the role of the integral and the parameter in the original system is presented. The new system is then analyzed by means of describing its symmetry and measure preservation. In Section 4 finding fixed points and periodic points (and their linear stability) and also computing the base points are discussed. These are done in the remaining subsections in Section 4. We have divided Section 4 into five subsections. This paper ends with some concluding remarks in Section 5.

\section{Formulation of the Problem}

Consider a three parameters family of partial difference equation on two dimensional lattice:

$\theta_{1}\left(V_{l, m+1} V_{l+1, m}-V_{l+1, m+1} V_{l, m}\right)+\theta_{2} V_{l+1, m+1} V_{l, m+1} V_{l+1, m} V_{l, m}=\theta_{3}$.

This equation is derived from the compatibility condition of the generalized Lax pair of the classical discrete sine-Gordon equation (see Zakaria and Tuwankotta, 2016). The travelling wave solutions of (1) can be obtained by considering the following form: 
International Journal of Mathematical, Engineering and Management Sciences

Vol. 5, No. 2, 363-377, 2020

https://doi.org/10.33889/IJMEMS.2020.5.2.030

$V_{l, m}=V_{n}, n=z_{1} l+z_{2} m$,

where $z_{1}$ and $z_{2}$ are relatively prime integers. Substituting this to (1), we have an infinite hierarchy of mapping labeled by $z_{1}$ and $z_{2}$, i.e.

$\theta_{1}\left(V_{n+z_{1}+z_{2}} V_{n}-V_{n+z_{1}} V_{n+z_{2}}\right)-\theta_{2} V_{n+z_{1}+z_{2}} V_{n+z_{2}} V_{n+z_{1}} V_{n}+\theta_{3}=0$.

If $z_{1}$ and $z_{2}$ are fixed, a mapping from $\mathbb{R}^{z_{1}+z_{2}} \rightarrow \mathbb{R}^{z_{1}+z_{2}}$ can be obtained from (2). It can be noted that by setting $\theta_{2}=\theta_{3}=1$ and $\theta_{1}=p q$ in (2), we can derive the two dimensional mappings in Quispel et al., (1991).

Let $z_{1}=z_{2}=1, \theta_{1}=\mu \theta_{2}$, and $\theta_{3}=\lambda \theta_{2}$. From (2), we derive:

$V_{n+2}=\frac{\lambda-\mu V_{n+1}^{2}}{V_{n}\left(V_{n+1}^{2}-\mu\right)}$,

which is a second order difference equation. Then, by writing $x_{n}=V_{n+1}$, and $y_{n}=V_{n}$.

We derive a system of first order difference equations:

$$
\begin{aligned}
& x_{n+1}=\frac{\lambda-\mu x_{n}^{2}}{y_{n}\left(x_{n}^{2}-\mu\right)}, \\
& y_{n+1}=x_{n} .
\end{aligned}
$$

Let us define: $f: \mathbb{R}^{2} \rightarrow \mathbb{R}^{2}$, by

$f(x, y)=\left(\frac{\left(\lambda-\mu x^{2}\right)}{y\left(x^{2}-\mu\right)}, x\right)$.

Using this, we can write (3) as:

$\left(x^{\prime}, y^{\prime}\right)=f(x, y)$

where the prime denotes the upshift. The mapping (4) has an integral, i.e.

$F(x, y)=\mu\left(\frac{x}{y}+\frac{y}{x}\right)-\left(x y+\lambda \frac{1}{x y}\right)$.

Thus, $F\left(x_{n+1}, y_{n+1}\right)=F\left(x_{n}, y_{n}\right)$ for all $n \in \mathbb{N}$.

\section{Reparametrized Mapping and Its Properties}

Consider the mapping in (4). For $\lambda=1$, we solve the equation $F(x, y)=0$ for $\mu$ to derive: 
International Journal of Mathematical, Engineering and Management Sciences

Vol. 5, No. 2, 363-377, 2020

https://doi.org/10.33889/IJMEMS.2020.5.2.030

$\mu(x, y)=\frac{\left(1+x^{2} y^{2}\right)}{\left(x^{2}+y^{2}\right)}$.

Substituting (6) into (4), we derive a new mapping:

$\left(x^{\prime}, y^{\prime}\right)=\hat{f}(x, y)=(-y, x)$,

with integral

$\hat{F}(x, y)=\frac{\left(1+x^{2} y^{2}\right)}{\left(x^{2}+y^{2}\right)}$.

Note that the integral (8) is nothing but the function $\mu(x, y)$ in (6). The new map, $\hat{f}(x, y)$, has the following properties:

- The orbits of $\hat{f}(x, y)$ is 4-periodic. This is simple to show that the linear map in (7) is trivial, its second iterate leads to $\left(x^{\prime}, y^{\prime}\right)=(-x,-y)$ thus all the results are immediate (the fourth iterate is the identity function).

- $\hat{f}(x, y)$ is area preserving. A two-dimensional map is area preserving (also called conservative) when its Jacobian determinant $|J(x, y)|$ is equal to 1 in all the points $(x, y)$ of the plane, which for the linear map in equation (7) is immediate (the Jacobian determinant is constant and equal to 1), as well as the so-called "reverse symmetry", since $\hat{f}^{-1}(u, v)=-\hat{f}(u, v)$. Alternatively, we can use different procedure to show that an integrable map is measure preserving (Roberts et al., 2002). For our map, $\hat{f}(x, y)$, the procedure can be followed as shown below.

The mapping $\hat{f}(x, y)$ is measure preserving (area preserving) because there is the density $\hat{\rho}(x, y)$ such that

$$
|D \hat{f}(x, y)|=\frac{\hat{\rho}(x, y)}{\hat{\rho}\left(x^{\prime}, y^{\prime}\right)}=1
$$

when the density $\hat{\rho}$ is given by

$$
\hat{\rho}(x, y)=\frac{1}{x y}\left[\frac{\partial F(x, y)}{\partial \mu}\right]^{-1}=\frac{1}{x^{2}+y^{2}} \text {. }
$$

- Consider $G_{1}(x, y)=(y, x)$. Note that $G_{1}\left(G_{1}(x, y)\right)=G_{1}(y, x)=(x, y)$. This implies that: $G_{1}^{-1}=G_{1}$. Since $G_{1} \circ \tilde{f} \circ G_{1}^{-1}=\tilde{f}^{-1}$ then $G_{1}$ is a reversing symmetry for $\hat{f}$.

- There exists a symmetry $S_{1}(x, y)=(-x,-y)$ such that $S_{1} \circ \hat{f} \circ S_{1}^{-1}=\hat{f}$.

The dynamics of the mappings in (7) for $\lambda=1$ on every level set $\hat{F}=c$ is basically identical to the dynamics of the mappings in (4) on the level set $F=0$ for $\mu=c$. Furthermore, this provides 
International Journal of Mathematical, Engineering and Management Sciences

Vol. 5, No. 2, 363-377, 2020

https://doi.org/10.33889/IJMEMS.2020.5.2.030

us with the existence of a 4-periodic points of the mappings: (7) for $\lambda=1$ for every $\mu$. The locations of these points are in the level set $F=0$.

Still fixing the value of $\lambda=1$, let us now, reparametrize the parameter in (4) by $\mu \rightarrow a_{0}+a_{1} \mu$. It follows immediately that the map is given by

$$
\tilde{f}(x, y)=\left(\frac{\left(1-\left(a_{0}+a_{1} \mu\right) x^{2}\right)}{y\left(x^{2}-\left(a_{0}+a_{1} \mu\right)\right)}, x\right) \text {. }
$$

Consequently, we transform the integral (5) to

$\tilde{F}(x, y)=\left(a_{0}+a_{1} \mu\right)\left(\frac{x}{y}+\frac{y}{x}\right)-\left(x y+\frac{1}{x y}\right)$.

For the case where: $a_{1} \neq 0$, let us add a constant in (10) which takes a special form: $b_{0}+b_{1} \mu$, i.e.

$$
\tilde{F}(x, y)=\left(a_{0}+a_{1} \mu\right)\left(\frac{x}{y}+\frac{y}{x}\right)-\left(x y+\frac{1}{x y}\right)+\left(b_{0}+b_{1} \mu\right) \text {, }
$$

Note that $\tilde{\tilde{F}}(x, y)$ is linear in $\mu$. Furthermore:

$$
\tilde{\tilde{F}} \circ \tilde{f}=\tilde{\tilde{F}}\left(\frac{\left(1-\left(a_{0}+a_{1} \mu\right) x^{2}\right)}{y\left(x^{2}-\left(a_{0}+a_{1} \mu\right)\right)}, x\right)=\tilde{\tilde{F}}(x, y) \text {. }
$$

This implies that we can look at the zero level curve of $\tilde{\tilde{F}}$ and solve it for $\mu$ :

$$
\mu=\tilde{\mu}(x, y)=\frac{1+x^{2} y^{2}-x^{2} a_{0}-y^{2} a_{0}-x y b_{0}}{x^{2} a_{1}+y^{2} a_{1}+x y b_{1}} \text {. }
$$

Substituting this expression of $\tilde{\mu}(x, y)$ into $\tilde{f}$ in (9) gives:

$$
\hat{\tilde{f}}(x, y)=\left(\frac{a_{1}\left(y-x^{4} y+x^{3} b_{0}\right)+x\left(1-x^{2} a_{0}\right) b_{1}}{a_{1}\left(-1+x^{4}+x y b_{0}\right)+x y\left(x^{2}-a_{0}\right) b_{1}}, x\right) \text {. }
$$

It is interesting to note that $\tilde{\mu}(\hat{\tilde{f}}(x, y))=\tilde{\mu}(x, y)$ which implies that $\tilde{\mu}(x, y)$ is an integral for the system:

$$
\left(x^{\prime}, y^{\prime}\right)=\hat{\tilde{f}}(x, y) \text {. }
$$

The mapping (13) has some properties:

- $\quad \hat{\tilde{f}}$ is measure preserving and orientation-reversing (or anti-measure preserving), which means (Roberts et al., 2002) 


$$
|D \hat{\tilde{f}}|=-\frac{\hat{\rho}(x, y)}{\hat{\rho}\left(\frac{a_{1}\left(y-x^{4} y+x^{3} b_{0}\right)+x\left(1-x^{2} a_{0}\right) b_{1}}{a_{1}\left(-1+x^{4}+x y b_{0}\right)+x y\left(x^{2}-a_{0}\right) b_{1}}, x\right)} .
$$

Note that from the right-hand side, the determinant of the Jacobian of $\hat{\tilde{f}}$ is

$$
\frac{b_{1}^{2} x^{2}\left(a_{0}^{2} x^{2}-a_{0}\left(x^{4}+1\right)+x^{2}\right)+a_{1} b_{0} b_{1} x^{2}\left(-2 a_{0} x^{2}+x^{4}+1\right)+a_{1}^{2}\left(\left(b_{0}^{2}-2\right) x^{4}+x^{8}+1\right)}{\left(b_{1} x y\left(x^{2}-a_{0}\right)+a_{1}\left(b_{0} x y+x^{4}-1\right)\right)^{2}} .
$$

Meanwhile, from the right-hand side, we have

$$
\begin{gathered}
-\left(\frac{\left(b_{1}^{2} x^{2}\left(a_{0}-x^{2}\right)\left(a_{0} x^{2}-1\right)\right)}{\left(a_{1}\left(-1+x^{4}+x y b_{0}\right)+x y\left(x^{2}-a_{0}\right) b_{1}\right)^{2}}+\frac{\left(a_{1} b_{0} b_{1} x^{2}\left(-2 a_{0} x^{2}+x^{4}+1\right)\right)}{\left(a_{1}\left(-1+x^{4}+x y b_{0}\right)+x y\left(x^{2}-a_{0}\right) b_{1}\right)^{2}}\right. \\
\left.+\frac{\left(a_{1}^{2}\left(\left(b_{0}^{2}-2\right) x^{4}+x^{8}+1\right)\right)}{\left(a_{1}\left(-1+x^{4}+x y b_{0}\right)+x y\left(x^{2}-a_{0}\right) b_{1}\right)^{2}}\right)
\end{gathered}
$$

where the so-called density $\hat{\rho}$ is given by

$$
\hat{\rho}(x, y)=\frac{1}{x y}\left(\left[\partial_{\mu} \tilde{\tilde{F}}\right]^{-1}\right)=\frac{1}{a_{1}\left(x^{2}+y^{2}\right)+b_{1} x y} \text {. }
$$

- The function $\hat{G}_{1}(x, y)=(-y,-x)$, is a reversing symmetry for $\hat{\tilde{f}}$.

\section{Critical Point and Base Point of the Integral}

There are two important elements in analyzing the dynamics of system (13), i.e. Fixed Point (FP) and Base Point (BP). FP can be obtained by finding the critical point of the integral function, while $\mathrm{BP}$ is defined as the point where the integral function is singular. At the BP, level sets for various values of the integral function intersect each other.

\subsection{The Critical Point}

The critical points of the integral function (12) are solutions of

$$
\begin{aligned}
& \frac{\partial \hat{\mu}}{\partial x}=\frac{a_{1}\left(2 x\left(y^{4}-1\right)+y\left(x^{2}-y^{2}\right) b_{0}\right)+y\left(x^{2} y^{2}-1+\left(-x^{2}+y^{2}\right) a_{0}\right) b_{1}}{\left(\left(x^{2}+y^{2}\right) a_{1}+x y b_{1}\right)^{2}}=0, \\
& \frac{\partial \hat{\mu}}{\partial y}=\frac{a_{1}\left(2 y\left(x^{4}-1\right)+\left(-x^{3}+x y^{2}\right) b_{0}\right)+x\left(x^{2} y^{2}-1+\left(x^{2}-y^{2}\right) a_{0}\right) b_{1}}{\left(\left(x^{2}+y^{2}\right) a_{1}+x y b_{1}\right)^{2}}=0 .
\end{aligned}
$$


International Journal of Mathematical, Engineering and Management Sciences

Vol. 5, No. 2, 363-377, 2020

https://doi.org/10.33889/IJMEMS.2020.5.2.030

To obtain the solutions, we can do as follow:

$$
y \frac{\partial \hat{\mu}}{\partial x}-x \frac{\partial \hat{\mu}}{\partial y}=0 \Leftrightarrow \frac{\left(x^{2}-y^{2}\right)\left(\left(x^{2}+y^{2}\right) a_{1}\left(2 x y-b_{0}\right)+\left(x^{2} y^{2}-\lambda+\left(x^{2}+y^{2}\right) a_{0}\right) b_{1}\right)}{\left(x^{2}+y^{2}\right) a_{1}+x y b_{1}}=0
$$

From the left-hand side in the last equation, we have two lines, $y=x$ and $y=-x$ as solutions. By substituting these into $\frac{\partial \hat{\mu}}{\partial x}=0$ or $\frac{\partial \hat{\mu}}{\partial y}=0$ and then solve it, we have $(x, y)=( \pm 1, \pm 1)$ and $( \pm 1, \mp 1)$

It is easy to verify that: $(1,1)$ and $(-1,-1)$ are two fixed points while $(1,-1)$ and $(-1,1)$ are two 2-periodic points.

\subsection{The Base Point}

Apart from the critical points, the so-called base points also play a crucial role in the dynamics of (13). Note that the invariant (12) can be written as a rational function. A point $\left(x_{0}, y_{0}\right)$ is a base point if it is a common zero of the numerator and denominator of $\tilde{\mu}(x, y)$ equal to zero. In our case, we will discuss two conditions, $a_{1}=0$ and $a_{1} \neq 0$.

For $a_{1}=0$, the base points are

$$
\left(x_{0}, y_{0}\right)=\left\{\left(0, \sqrt{\frac{1}{a_{0}}}\right),\left(0,-\sqrt{\frac{1}{a_{0}}}\right),\left(\sqrt{\frac{1}{a_{0}}}, 0\right),\left(-\sqrt{\frac{1}{a_{0}}}, 0\right)\right\}
$$

Note that the points in eq. (15) are base points of the system (13) when the parameter $a_{1}=0$. Two base points, $\left( \pm \sqrt{\frac{1}{a_{0}}}, 0\right)$, are directly mapped to infinity by the mapping $\hat{\tilde{f}}$, in the sense that one of both of the component of $\hat{\tilde{f}}$ blows up at these points. And then the other points, $\left(0, \pm \sqrt{\frac{1}{a_{0}}}\right)$, are base points in which they are mapped to other base points after one iterate of $\hat{\tilde{f}}$.

For $a_{1} \neq 0$, the base points can be obtained by solving $\left(1+x^{2} y^{2}-x^{2} a_{0}-y^{2} a_{0}-x y b_{0}\right)=0$ and $\left(x^{2} a_{1}+y^{2} a_{1}+x y b_{1}\right)=0$. Based on our computations, the base points are $\left(x_{0}, y_{0}\right)=\left\{\left( \pm x_{10}, \pm y_{10}\right),\left(\mp x_{10}, \pm y_{10}\right),\left( \pm x_{20}, \pm y_{20}\right),\left(\mp x_{20}, \pm y_{20}\right)\right\}$

where 
International Journal of Mathematical, Engineering and Management Sciences

Vol. 5, No. 2, 363-377, 2020

https://doi.org/10.33889/IJMEMS.2020.5.2.030

$x_{10}=-\sqrt{\frac{\sqrt{(\gamma+d)^{2}\left(b_{0}-a_{0} d\right)^{2}-8\left(\gamma d+d^{2}-2\right)}+a_{0} \gamma d+a_{0} d^{2}-b_{0} \gamma-b_{0} d}{2\left(\gamma d+d^{2}-2\right)}} ;$
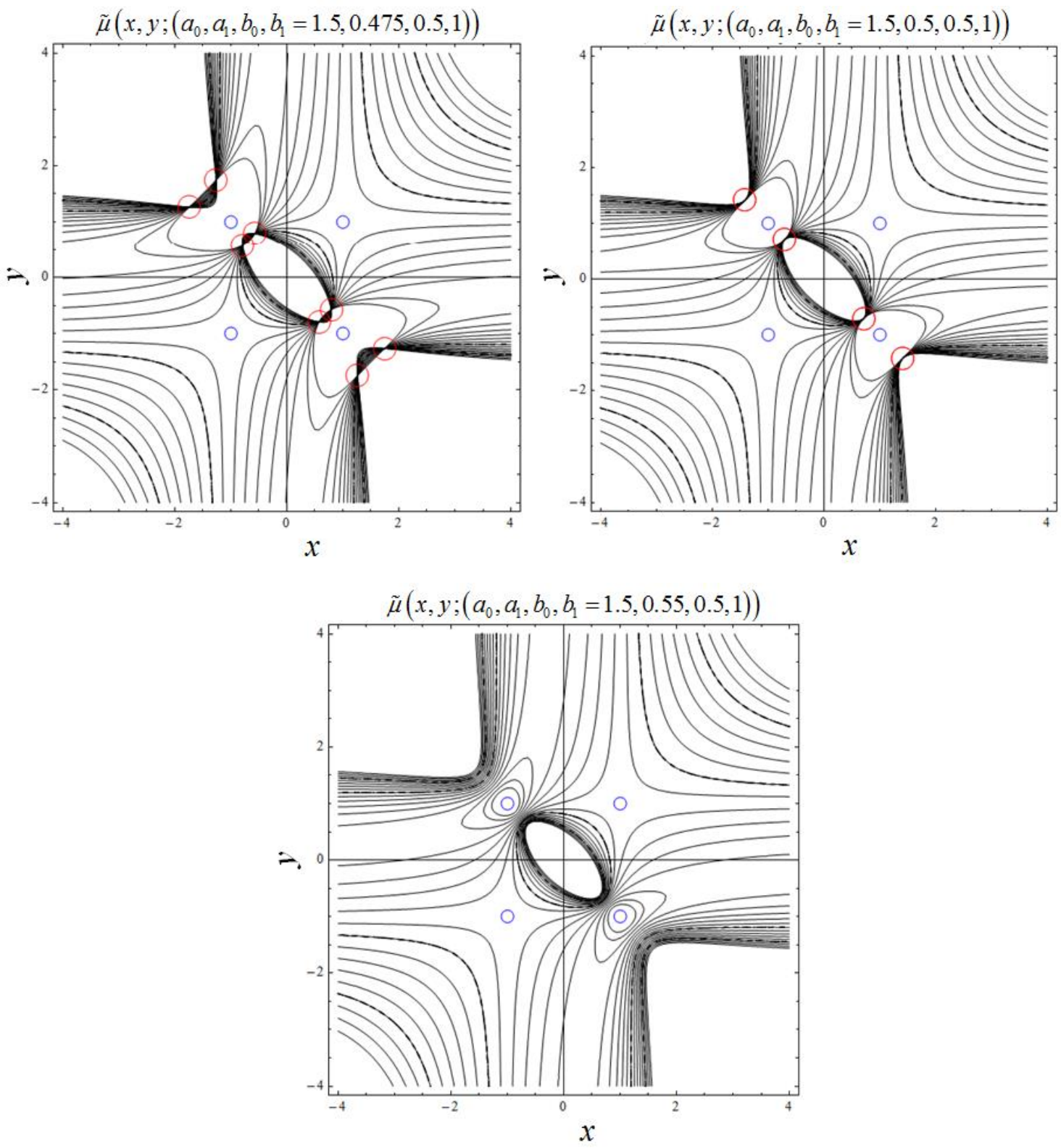

Figure 1. The level sets, the fixed points, the 2-periodic points, and the real base points of the integral (12) for parameter values $\left(a_{0}, a_{1}, b_{0}, b_{1}\right)=(1.5,0.475,0.5,1)$ (the diagram in the first row-left), $\left(a_{0}, a_{1}, b_{0}, b_{1}\right)=$ $(1.5,0.5,0.5,1)$ (the diagram in the first row-right), and $\left(a_{0}, a_{1}, b_{0}, b_{1}\right)=(1.5,0.55,0.5,1)$ (the diagram in the second row). 
International Journal of Mathematical, Engineering and Management Sciences

Vol. 5, No. 2, 363-377, 2020

https://doi.org/10.33889/IJMEMS.2020.5.2.030

$y_{10}=-\frac{x_{10}}{2}(d+\gamma)$

$x_{20}=-\sqrt{\frac{\sqrt{(d-\gamma)^{2}\left(b_{0}-a_{0} d\right)^{2}+8 \gamma d-8 d^{2}+16}-a_{0} \gamma d+a_{0} d^{2}+b_{0} \gamma-b_{0} d}{2\left(-\gamma d+d^{2}-2\right)}} ;$

$y_{20}=-\frac{x_{20}}{2}(d-\gamma) ; \quad d=\frac{b_{1}}{a_{1}}, a_{1} \neq 0 ; \quad \gamma=\sqrt{d^{2}-4}$.

All base points depend on the parameters $a_{0}, a_{1}, b_{0}$, and $b_{1}$. To obtain the real base points for $\left(x_{0}, y_{0}\right)=\left\{\left( \pm x_{10}, \pm y_{10}\right),\left(\mp x_{10}, \pm y_{10}\right)\right\}$, the parameters should satisfy the following conditions $b_{0} \in \mathbb{R} \wedge a_{0} \geq \frac{b_{0}}{d}+2 \sqrt{\frac{2 \gamma d+2 d^{2}-4}{d^{2}(\gamma+d)^{2}}} \wedge \gamma>0 \wedge\left(\left(d+2<0 \wedge \gamma+d<\frac{2}{d}\right) \vee d>2\right)$.

And then to obtain the real base points for $\left(x_{0}, y_{0}\right)=\left\{\left( \pm x_{20}, \pm y_{20}\right),\left(\mp x_{20}, \pm y_{20}\right)\right\}$, the parameters should satisfy the following conditions

$$
\begin{aligned}
b_{0} \in \mathbb{R} & \wedge\left(d<-2 \wedge \gamma>0 \wedge a_{0} \geq \frac{b_{0}}{d}+2 \sqrt{-\frac{2 \gamma d-2 d^{2}+4}{d^{2}(\gamma-d)^{2}}}\right) \\
& \vee\left(d>2 \wedge 0<\gamma<\frac{d^{2}-2}{d} \wedge a_{0} \geq \frac{b_{0}}{d}+2 \sqrt{-\frac{2 \gamma d-2 d^{2}+4}{d^{2}(\gamma-d)^{2}}}\right)
\end{aligned}
$$

Figure 1 shows the base points (red-circle) together with fixed points and 2-periodic points (blue-circle). Three diagrams in Figure 1 are some level sets of integral (12) together with the fixed points, the 2-periodic points, and the base points for $a_{0}=1.5$. The diagram has fixed parameter values $\left(a_{1}, b_{0}, b_{1}\right)=(0.475,0.5,1.0)$ (up-left diagram), $\left(a_{1}, b_{0}, b_{1}\right)=(0.5,0.5,1.0)$ (up-right diagram) and $\left(a_{1}, b_{0}, b_{1}\right)=(0.55,0.5,1.0)$ (down diagram).

\subsection{Preimages of the Base Points}

A base point is mapped to infinity by the mapping $\hat{\tilde{f}}$. Furthermore, there are two base points which do not refer to a base point. But after one iteration they are mapped to base points. This means that if we start at those points, the iteration of $\hat{\tilde{f}}$ will be sent to infinity after two iterations. Let us name the set of points which are mapped by $\hat{\tilde{f}}$ to a base point by $P_{1}$. We can then look at the set of points which are mapped by $\hat{\tilde{f}}$ into $P_{1}$, or the preimage of $P_{1}$ under $\hat{\tilde{f}}$; and name the 
International Journal of Mathematical, Engineering and Management Sciences

Vol. 5, No. 2, 363-377, 2020

https://doi.org/10.33889/IJMEMS.2020.5.2.030

set $P_{2}$. Continuing in a similar way, we constructed $P_{3}, P_{4}$ and so on. Thus, the dynamical system (13) is well defined if we exclude the points in $\bigcup_{1}^{\infty} P_{k}$.

Let us present a few explicit computations of $P_{k}, k=1,2,3$ as examples.

Consider the situation where $(x, y)=\left(-\frac{1}{\sqrt{a_{0}}}, 0\right)$ for $a_{0}>0$ and denote the system (13) by

$\hat{\tilde{f}} \quad: \quad \mathbb{R}^{2} \rightarrow \mathbb{R}^{2}$

$(x, y) \mapsto\left(\frac{a_{1}\left(y\left(-x^{4}+1\right)+x^{3} b_{0}\right)+x\left(1-x^{2} a_{0}\right) b_{1}}{a_{1}\left(x^{4}-1+x y b_{0}\right)+x y\left(x^{2}-a_{0}\right) b_{1}}, x\right)$.

The preimage of $(x, y)=\left(-\frac{1}{\sqrt{a_{0}}}, 0\right)$ by $(16)$ is the solution of the following system

$$
\begin{aligned}
x & =0 \\
\frac{a_{1}\left(y\left(-x^{4}+1\right)+x^{3} b_{0}\right)+x\left(1-x^{2} a_{0}\right) b_{1}}{a_{1}\left(x^{4}-1+x y b_{0}\right)+x y\left(x^{2}-a_{0}\right) b_{1}} & =-\frac{1}{\sqrt{a_{0}}} .
\end{aligned}
$$

The solution is

$$
P_{1}=\hat{\tilde{f}}^{-1}\left(\left\{\left(-\frac{1}{\sqrt{a_{0}}}, 0\right)\right\}\right)=\left\{\left(0, \frac{1}{\sqrt{a_{0}}}\right) \mid a_{0}>0\right\} \text {. }
$$

For the preimages $P_{2}$ and $P_{3}$, we have

$$
\begin{aligned}
& P_{2}=\hat{\tilde{f}}^{-1}\left(\left\{\left(0, \frac{1}{\sqrt{a_{0}}}\right)\right\}\right)=\left\{\left(\sigma, \frac{\sigma^{3} b_{0}}{\sigma^{4}-1}\right) \mid b_{0} \in \mathbb{R}\right\}, \\
& P_{3}=\hat{\tilde{f}}^{-1}\left(\left\{\left(\sigma, \frac{\sigma^{3} b_{0}}{\sigma^{4}-1}\right)\right\}\right)=\left\{\left(\frac{\sigma^{3} b_{0}}{\sigma^{4}-1},-\frac{\sigma\left(\left(\sigma^{4}-1\right)^{2}-\sigma^{4} b_{0}^{2}\right)}{\left(\sigma^{4}-1\right)^{2}-\sigma^{8} b_{0}^{2}}\right) \mid \sigma>0, b_{0} \in \mathbb{R}\right\},
\end{aligned}
$$

where $\sigma=\frac{1}{\sqrt{a_{0}}}>0$.

The graphs of $P_{2}$ to $P_{6}$ for $a_{0}=4$ are presented in Figure 2. The graph of $P_{2}, P_{3}, P_{4}, P_{5}$, and $P_{6}$ are plotted using green, red, blue, orange, and black, respectively. It is interesting to note that if 
International Journal of Mathematical, Engineering and Management Sciences

Vol. 5, No. 2, 363-377, 2020

https://doi.org/10.33889/IJMEMS.2020.5.2.030

we fix to one of the level sets of the integral, an preliminary observation shows an indication that there are only finitely many intersection points between $\bigcup_{1}^{6} P_{k}$. Whether it is true when $\bigcup_{1}^{\infty} P_{k}$ is a subject of future investigation.
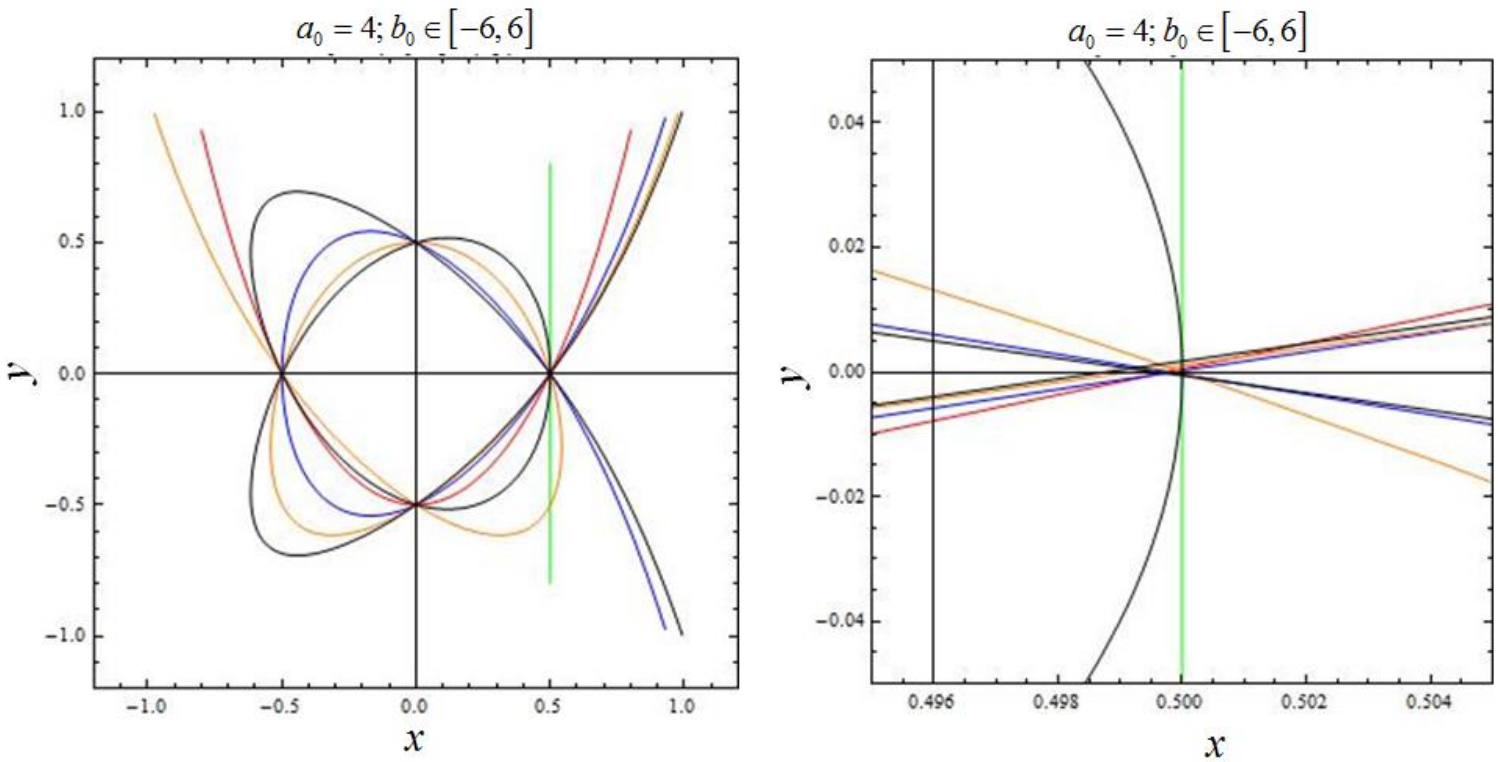

Figure 2. Plotting of the curves $P_{2} \ldots P_{6}$ for $a_{0}=4$. We see that all curves pass through all base points (left).

Enlarged view of the left image around a point $(1 / 2,0)$ to see the finer structure of the graph (right).

\subsection{Generic Situation}

To study the dynamics of the system (13), we have plotted some of the level sets of the integral (see Figure 3). These level sets contain solution of the system (13). Furthermore, by studying how the level sets deformed as we vary the value parameter, the bifurcations in the system can be studied. This is however beyond the scope of this paper.

The two diagrams in the upper part of Figure 3 are some the level sets of integral (12) for $a_{0}=4$. The diagram on the left-hand side has fixed $\left(a_{1}, b_{0}, b_{1}\right)=(0.7,0.5,1.0)$, while for the diagram on the right-hand side is $\left(a_{1}, b_{0}, b_{1}\right)=(-0.875,0.5,1.0)$. In the Figure 3 (down), we plot $a_{0}=4$, $\left(b_{0}, b_{1}\right)=(0.5,1.0)$, and $a_{1} \in(-3,0)$ around a fixed point $(x, y)=(-1,1)$ (left); and we plot $a_{0}=4,\left(b_{0}, b_{1}\right)=(0.5,1.0)$, and $a_{1} \in(-1,2)$ around the fixed point $(x, y)=(1,1)$ (right).

By setting $a_{0}=4$ and $a_{1}=0.7$, we have all of the curves around the fixed points, $(1,1)$ and $(-1,-1)$, are of hyperbolic type. On the other hand we have all of the curves around the 2-periodic points, $(-1,1)$ and $(1,-1)$, are of elliptic type. But for $a_{0}=4$ and $a_{1}=-0.875$, the situation is reverse, i.e. all of the curves around the fixed points are of elliptic type while all of the curves around the 2-periodic points are of hyperbolic type. 
International Journal of Mathematical, Engineering and Management Sciences

Vol. 5, No. 2, 363-377, 2020

https://doi.org/10.33889/IJMEMS.2020.5.2.030
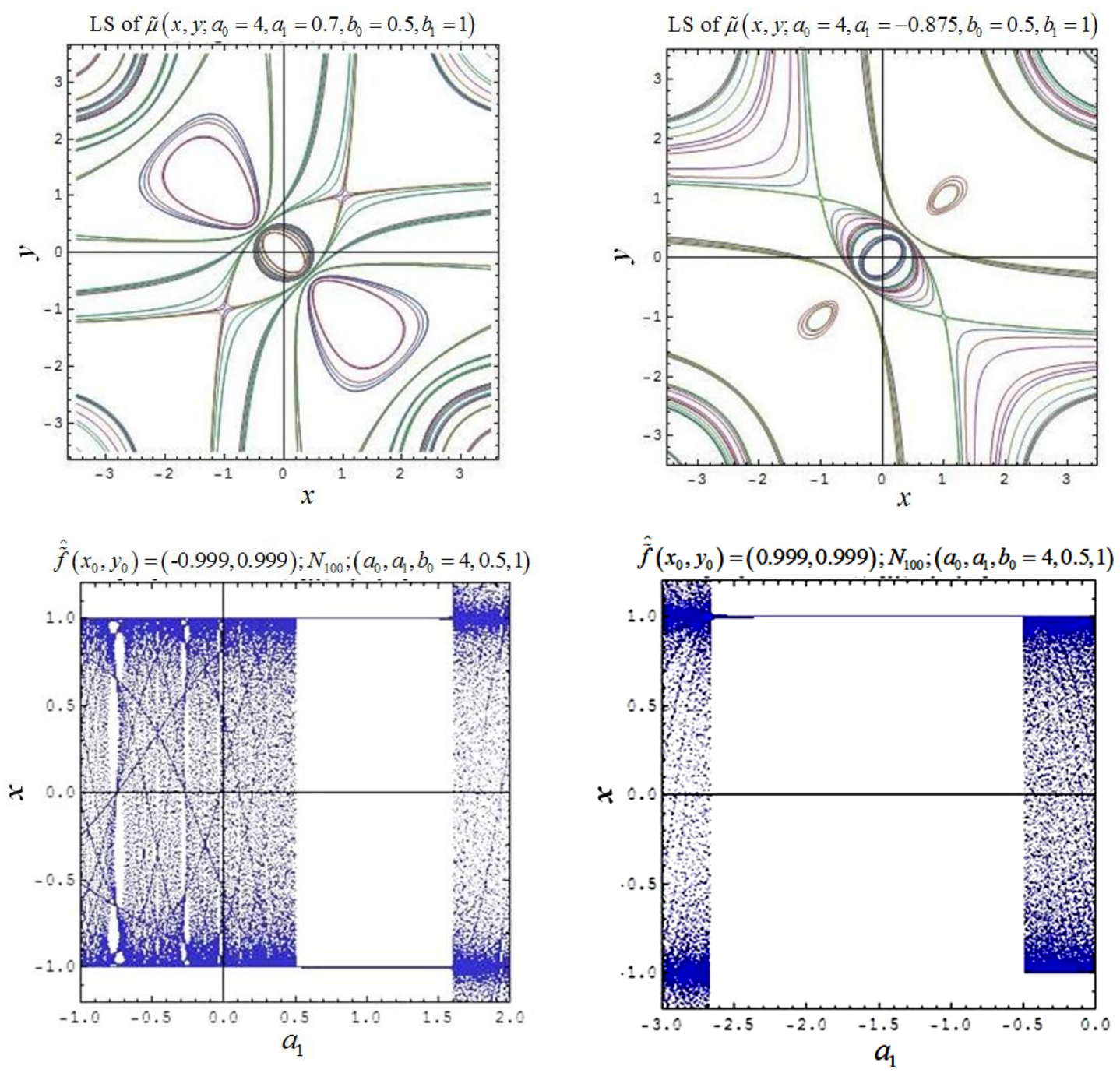

Figure 3. The level set forms of the integral (12) for the parameter value $a_{0}=4$ (up). Bifurcation situation corresponds with its (down).

\subsection{Stability}

The stability of the system in the vicinity of the fixed points can be extracted from the integral function (see Kulenovic and Merino, 2002). In this case, we use the concept of Lyapunov stability to obtain the information of the stability of system (13). Note that the Hessian matrix evaluated at the fixed point $\left(x^{*}, y^{*}\right)=( \pm 1, \pm 1)$ is 
International Journal of Mathematical, Engineering and Management Sciences

Vol. 5, No. 2, 363-377, 2020

https://doi.org/10.33889/IJMEMS.2020.5.2.030

$H=\left(\begin{array}{ll}A & B \\ B & A\end{array}\right)$

where

$$
\begin{aligned}
& A=\frac{2\left(a_{1} b_{0}-\left(a_{0}-1\right) b_{1}\right)}{\left(2 a_{1}+b_{1}\right)^{2}} ; \\
& B=\frac{2\left(-a_{1}\left(-4+b_{0}\right)+\left(1+a_{0}\right) b_{1}\right)}{\left(2 a_{1}+b_{1}\right)^{2}} .
\end{aligned}
$$

The determinant of the Hessian matrix $H$ is

$\operatorname{Det}(H)=-\frac{4\left(a_{1}\left(8-4 b_{0}\right)+4 a_{0} b_{1}\right)}{\left(2 a_{1}+b_{1}\right)^{3}}$

If the determinant of Hessian matrix $H$ is positive then the integral (12) attains a minimum at $\left(x^{*}, y^{*}\right)=( \pm 1, \pm 1)$. In Table 1 , we have listed the condition for the value of the parameters of the system, so that the determinant of $H$ is positive. Consequently, the fixed points of (13), $\left(x^{*}, y^{*}\right)=( \pm 1, \pm 1)$, are the centre points (stable).

Table 1. The conditions for the value of the parameters so that the determinant of $H$ is positive

\begin{tabular}{|c|c|c|c|c|}
\hline No. & $a_{0}$ & $b_{1}$ & $b_{0}$ & $a_{1}$ \\
\hline 1. & $a_{0}>0$ & $b_{1}>0$ & $2-2 a_{0}<b_{0}<2$ & $\frac{a_{0} b_{1}}{-2+b_{0}}<a_{1}<-\frac{b_{1}}{2}$ \\
\hline 2. & $a_{0}>0$ & $b_{1}>0$ & $b_{0}<2-2 a_{0}$ & $-\frac{b_{1}}{2}<a_{1}<\frac{a_{0} b_{1}}{-2+b_{0}}$ \\
\hline 3. & $a_{0}>0$ & $b_{1}>0$ & $b_{0}>2$ & $a_{1}<-\frac{b_{1}}{2} \vee a_{1}>\frac{a_{0} b_{1}}{-2+b_{0}}$ \\
\hline 4. & $a_{0}<0$ & $b_{1}>0$ & $2<b_{0} \leq 2-2 a_{0}$ & $a_{1}>-\frac{b_{1}}{2} \vee a_{1}<\frac{a_{0} b_{1}}{-2+b_{0}}$ \\
\hline 5. & $a_{0}<0$ & $b_{1}>0$ & $b_{0}>2-2 a_{0}$ & $a_{1}>\frac{a_{0} b_{1}}{-2+b_{0}} \vee a_{1}<-\frac{b_{1}}{2}$ \\
\hline 6. & $a_{0}<0$ & $b_{1}>0$ & $b_{0}<2$ & $-\frac{b_{1}}{2}<a_{1}<\frac{a_{0} b_{1}}{-2+b_{0}}$ \\
\hline 7. & $a_{0}>0$ & $b_{1}<0$ & $2-2 a_{0}<b_{0}<2$ & $-\frac{b_{1}}{2}<a_{1}<\frac{a_{0} b_{1}}{-2+b_{0}}$ \\
\hline
\end{tabular}


International Journal of Mathematical, Engineering and Management Sciences

Vol. 5, No. 2, 363-377, 2020

https://doi.org/10.33889/IJMEMS.2020.5.2.030

Table 1 continued...

\begin{tabular}{|c|c|c|c|c|}
\hline 8. & $a_{0}>0$ & $b_{1}<0$ & $b_{0}>2$ & $a_{1}>-\frac{b_{1}}{2}$ \\
\hline 9. & $a_{0}>0$ & $b_{1}<0$ & $b_{0}<2-2 a_{0}$ & $\frac{a_{0} b_{1}}{-2+b_{0}}<a_{1}<-\frac{b_{1}}{2}$ \\
\hline 10. & $a_{0}<0$ & $b_{1}<0$ & $2<b_{0} \leq 2-2 a_{0}$ & $a_{1}<-\frac{b_{1}}{2} \quad \vee \quad a_{1}>\frac{a_{0} b_{1}}{-2+b_{0}}$ \\
\hline 11. & $a_{0}<0$ & $b_{1}<0$ & $b_{0}>2-2 a_{0}$ & $a_{1}>-\frac{b_{1}}{2} \quad \vee \quad a_{1}<\frac{a_{0} b_{1}}{-2+b_{0}}$ \\
\hline 12. & $a_{0}<0$ & $b_{1}<0$ & $b_{0}<2$ & $-\frac{a_{0} b_{1}}{-2+b_{0}}<a_{1}<-\frac{b_{1}}{2}$ \\
\hline 13. & $a_{0}=0$ & $b_{1}<0$ & $b_{0}<2$ & $0<a_{1}<-\frac{b_{1}}{2}$ \\
\hline 14. & $a_{0}=0$ & $b_{1}<0$ & $b_{0}>2$ & $a_{1}>-\frac{b_{1}}{2} \quad \vee \quad a_{1}<0$ \\
\hline 15. & $a_{0}=0$ & $b_{1}>0$ & $b_{0}<2$ & $-\frac{b_{1}}{2}<a_{1}<0$ \\
\hline 16. & $a_{0}=0$ & $b_{1}>0$ & $b_{0}>2$ & $a_{1}>0 \quad \vee \quad a_{1}<-\frac{b_{1}}{2}$ \\
\hline 17. & $a_{0} \in \mathbb{R}$ & $b_{1}=0$ & $b_{0} \neq 2$ & $a_{1}>0$ \\
\hline 18. & $a_{0}<0$ & $b_{1} \neq 0$ & $b_{0} \in \mathbb{R}$ & $a_{1}=0$ \\
\hline
\end{tabular}

\section{Conclusion}

After re-parametrizing the parameter in (9) and in (10) by $\mu \rightarrow a_{0}+a_{1} \mu$ and $\tilde{F} \rightarrow \tilde{F}+\left(b_{0}+b_{1} \mu\right)$, we have a new mapping $\hat{\tilde{f}}$. The properties of this mapping are integrable, measure preserving, and reversible. Furthermore, it has two fixed points and two 2-periodic points which are of an elliptic type and a hyperbolic type. It is interesting to underline the fact that we have the set of $P_{k}$ consisting of points in $\mathbb{R}^{2}$ which is mapped to infinity after $k$-iterates of the map. Then we can consider the set of $\mathcal{P}=\bigcup_{1}^{\infty} P_{k}$. For an arbitrary level set of the integral (12), the question should be whether the intersection between the set of $\mathcal{P}$ with the level set is finite or infinite (could it be dense on the level set). This is a subject to future investigation.

\section{Conflict of Interest}

The authors confirm that there is no conflict of interest for this publication.

\section{Acknowledgements}


International Journal of Mathematical, Engineering and Management Sciences

Vol. 5, No. 2, 363-377, 2020

https://doi.org/10.33889/IJMEMS.2020.5.2.030

LZ acknowledges supports from a The Research and Community Services of Lampung University through Lampung University DIPA BLU Research Grants 2017. JMT research is supported by: Riset KK ITB 2017. The authors would like to thank you very much for the reviewer's comments/suggestions that improved the contents of the article.

\section{References}

Celledoni, E., Evripidou, C., McLaren, D.I., Owren, B., Quispel, G.R.W., \& Tapley, B.K. (2019). Discrete Darboux polynomials and the search for preserved measures and integrals of rational maps. Arxiv Preprint Arxiv: 1902.04685v1.

Duistermaat, J.J. (2010). Discrete integrable systems: QRT maps and elliptic surfaces. Springer Monographs in Mathematics, Springer-Verlag, New York.

Joshi, N., \& Kassotakis, P. (2019). Re-factorising a QRT map. Arxiv Preprint Arxiv:1906.00501v1[nlin.SI]

Kulenovic, M.R.S., \& Merino, O. (2002). Discrete dynamical systems and difference equations with mathematica. Chapman and Hall/CRC, Boca Raton, Florida. USA.

Quispel, G.R.W., Capel, H.W, \& Roberts, J.A.G. (2005). Duality for discrete integrable systems.Journal of Physics A: Mathematical and General, 38(18), 3965.

Quispel, G.R.W., Capel, H.W, Papageorgiou V.G, \& Nijhoff, F.W (1991). Integrable mappings derived from soliton equations. Physica A: Statistical Mechanics and its Applications, 173(1-2), 243-266.

Quispel, G.R.W., Roberts, J.A.G., \& Thompson, C.J. (1988). Integrable mappings and soliton equations. Physics Letters A, 126(7), 419-421.

Quispel, G.R.W., Roberts, J.A.G., \& Thompson, C.J. (1989). Integrable mappings and soliton equations II. Physica D, 34(1-2), 183-192.

Roberts, J.A.G., Iatrou A., \& Quispel, G.R.W. (2002). Interchanging parameters and integrals in dynamical systems: the mapping case, Journal of Physics A: Mathematical and General, 35(9), 2309-2325.

Tuwankotta, J.M., Van der Kamp, P., Quispel, G.R.W., \& Saputra, K.V.I. (2019). Generating a chain of maps which preserve the same integral as a given map. arXiv Preprint arXiv:1902.05206.

Tuwankotta, J.M., Quispel G.R.W., \& Tamizhmani, K.M. (2004). Dynamics and bifurcations of a three-dimensional piecewise-linear integrable map. Journal of Physics A: Mathematical and General, 37(50), 12041.

Van der Kamp, P.H., \& Quispel, G.R.W. (2010). The staircase method: integrals for periodic reductions of integrable lattice equations. Journal of Physics A: Mathematical and Theoretical, 43(46) , 465207.

Van der Kamp, P.H., Rojas, O., \& Quispel, G.R.W. (2007). Closed-form expressions for integrals of mKdV and sine-Gordon maps. Journal of Physics A: Mathematical and Theoretical, 40(42), 12789.

Zakaria, L., \& Tuwankotta, J.M. (2016). Dynamics and bifurcations in a two-dimensional maps derived from a generalized $\Delta \Delta$ sine-Gordon equation. Far East Journal of Dynamical Systems, 28(3), 165-194. 